\title{
Zu Theodoros Bestons Enkomion auf die hl. Euphemia.
}

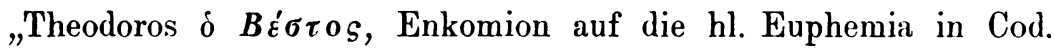
Sabbait. 10j saec. 14 fol. 208-226" lautet bei Ehrhard in Krumbachers G B L ${ }^{2}$ 176,10 die Notiz über den auch sonst unbekannten Panegyriker, dessen einziges Werk uns nun der gelehrte Mönch des Laura-Klosters Alex. Eumorphopulos aus Cod. Laur. $56 \Delta$ saec. $14 \mathrm{f} .166 \mathrm{ff}$. durch

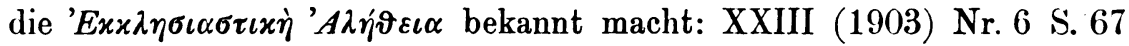
$-68 ; 7,81-83 ; 9,110-111 ; 11,139-140 ; 16,202-204 ; 22,259$ -260 und $32,345-346$.

Der Zuname des Verfassers lautet in Cod. Laur. $\beta \varepsilon \dot{\varepsilon} \tau \omega \nu$, wemn

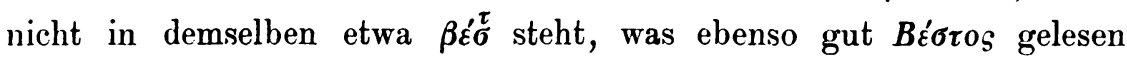
werden kann. Theodoros hat jede Andeutung auf seine eigene Person geflissentlich vermieden; auch irgendein anderes historisches oder topographisches Interesse wird man seinem Enkomion kaum abgewinnen können. Der griechische Wortschatz wird durch 13 Wörter bereichert, die im Thesaurus, bei Sophocles und bei Kumanudes fehlen:

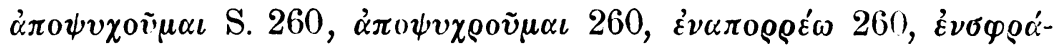

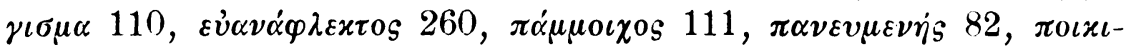

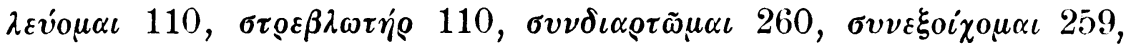

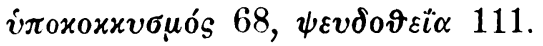

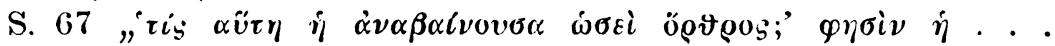

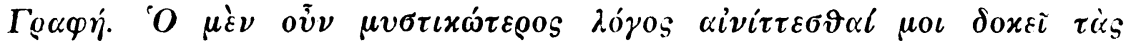

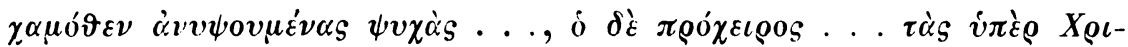

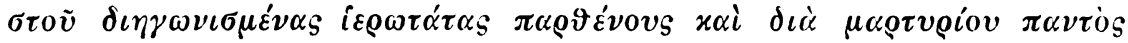

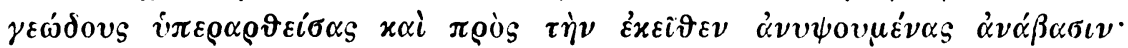

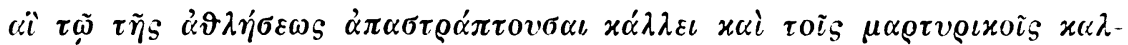

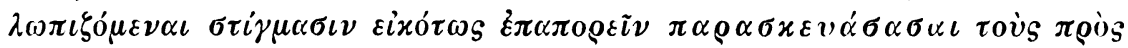

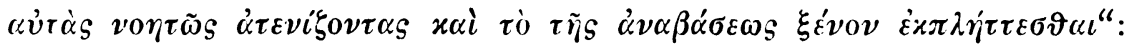
lies $\pi \alpha \rho \varepsilon \sigma x \varepsilon v \alpha \dot{\alpha} \alpha \sigma \iota$.

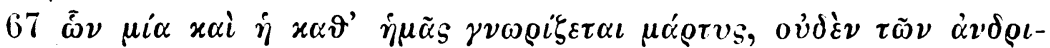

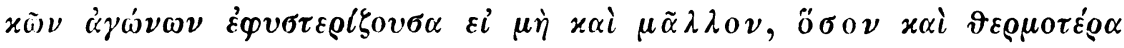

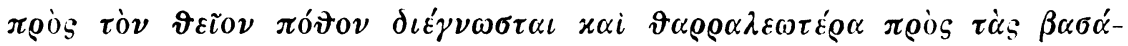

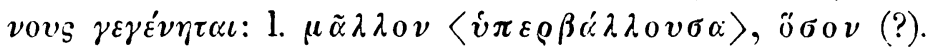




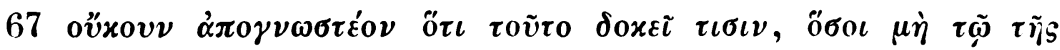

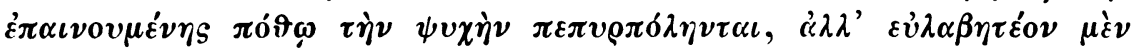

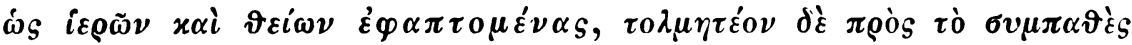

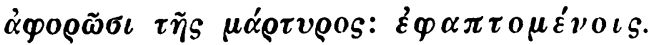

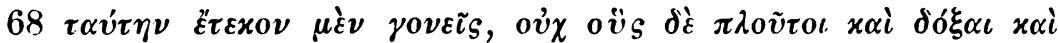

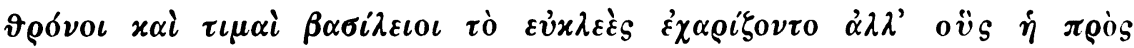

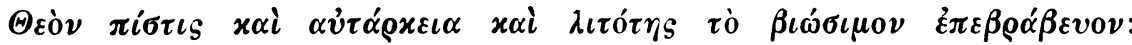
ois, oís.

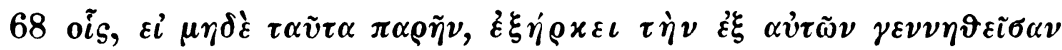

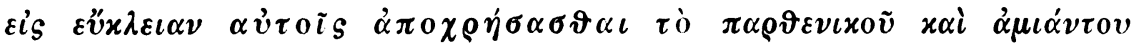

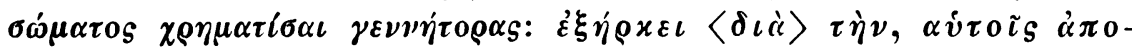

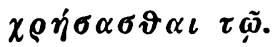

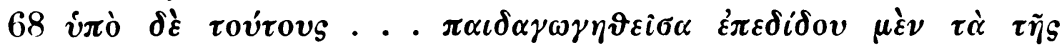

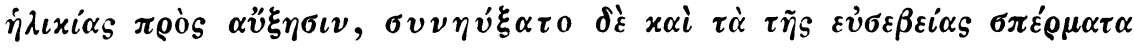

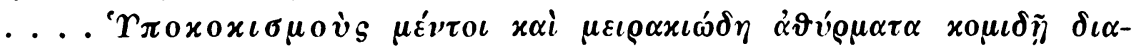

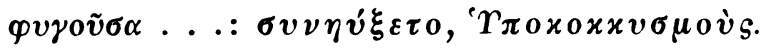

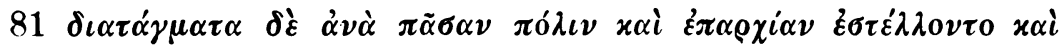

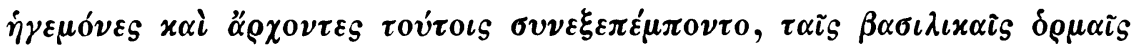

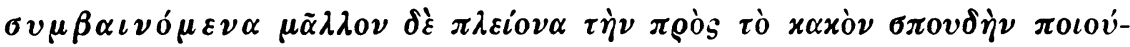
$\mu \varepsilon \nu o_{S} \tilde{\varepsilon} x \alpha \sigma \tau \sigma_{S}: \sigma v \mu \beta \alpha \iota \nu 0 \mu \varepsilon \dot{\varepsilon} \eta \nu$.

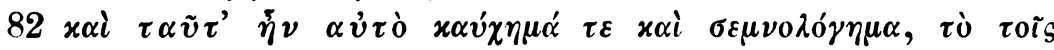

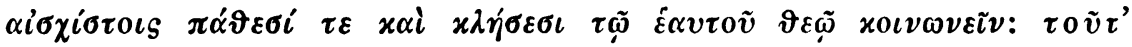
$\dot{\eta} v \alpha \dot{v} \tau \tilde{\varphi}$.

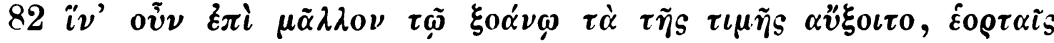

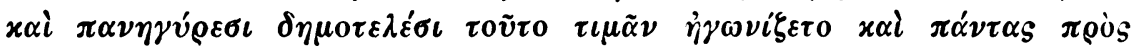

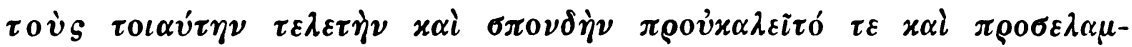
$\beta \alpha \dot{\nu \varepsilon \tau \tau: ~} \tau \dot{\eta} \nu$.

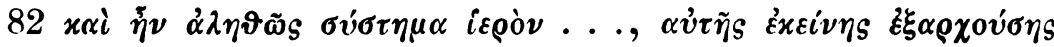

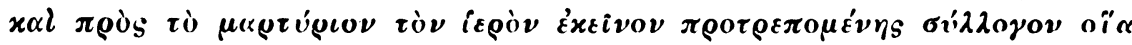

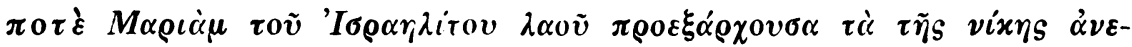

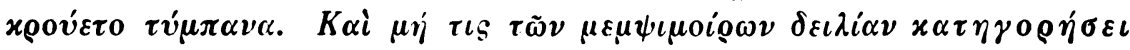

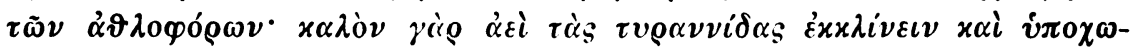

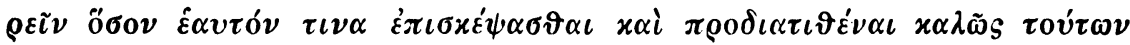

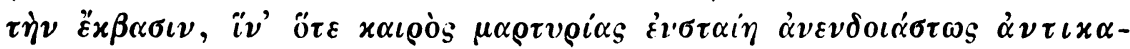

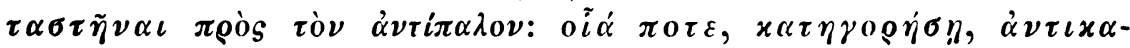
$\tau \alpha \sigma \tau \alpha i \eta$.

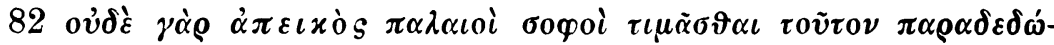

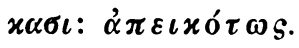

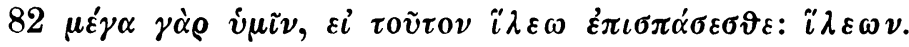

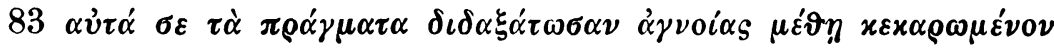

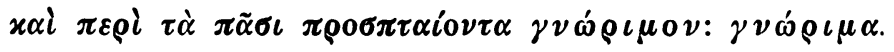




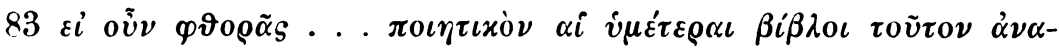

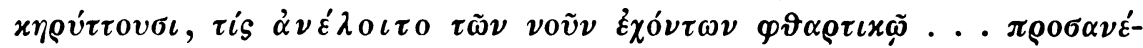

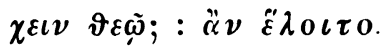

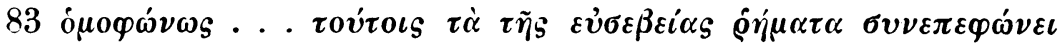

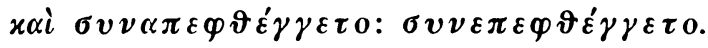

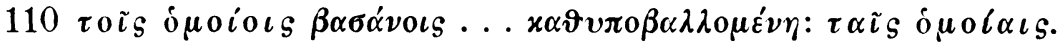

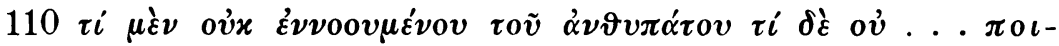

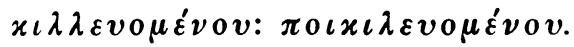

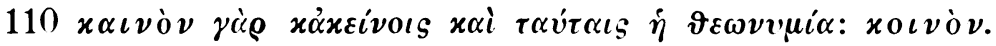

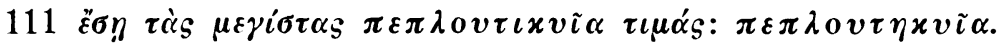

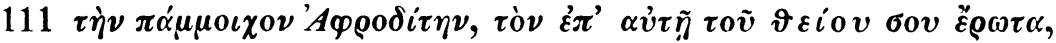

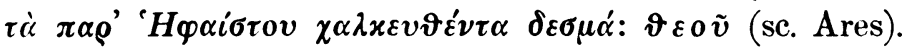

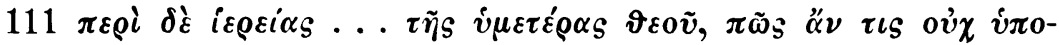

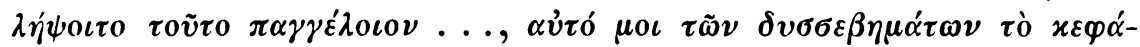

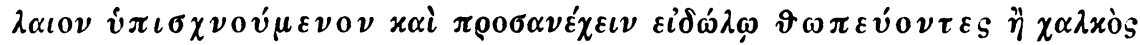

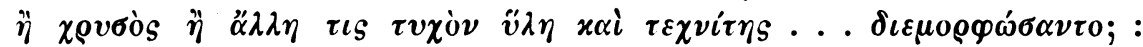

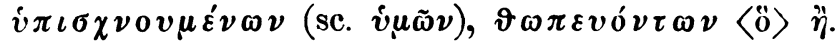

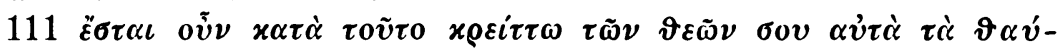

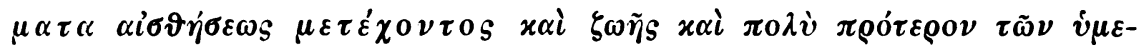

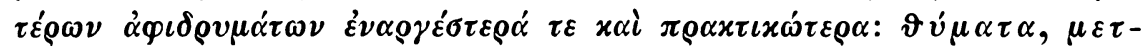
$\varepsilon \chi 0 \nu \tau \alpha$.

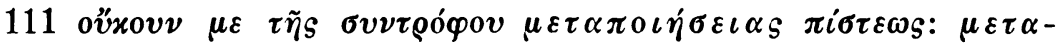

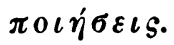

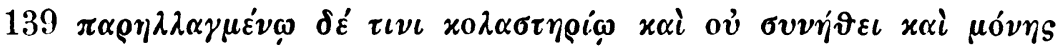

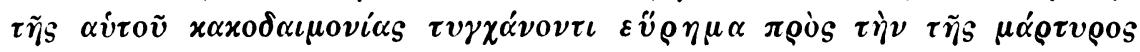

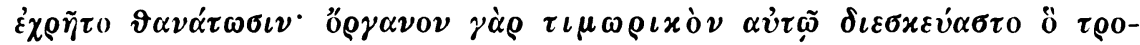

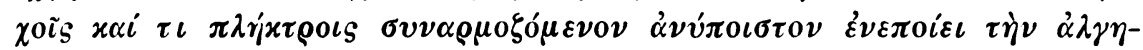

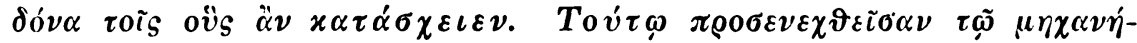
$\mu \alpha \tau \iota$ etc.: $\varepsilon \dot{v} \rho \eta \mu \alpha \tau \iota, \tau \iota \mu \omega \rho \eta \tau \iota x o ̀ \nu, \tau \iota \sigma \iota, x \alpha \tau \alpha^{\prime} \sigma \chi 0 \iota . \quad ' E v \tau o v ́ \tau \omega$.

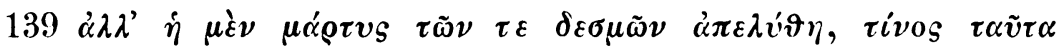

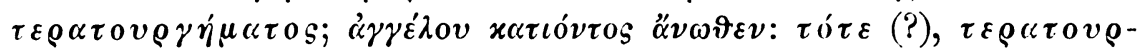
$\gamma \dot{\eta} \sigma \alpha \nu \tau o s$.

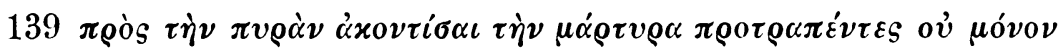

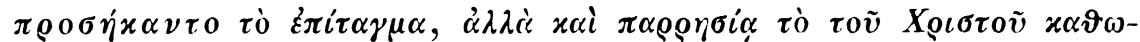

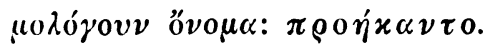

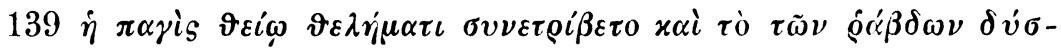

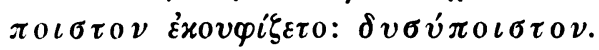

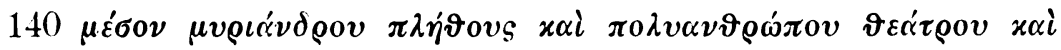

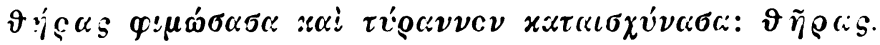

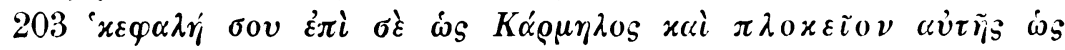


432 I. Abt. P. N. Papageorgiu: Zu Theod. Bestons Enkomion a. d. hl. Euphemia

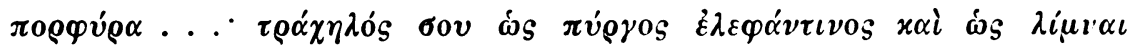

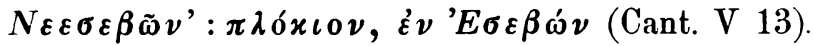

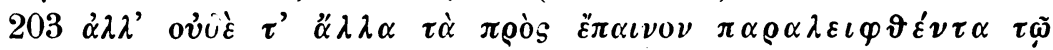

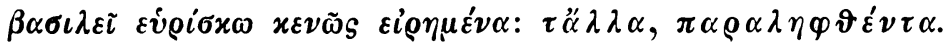

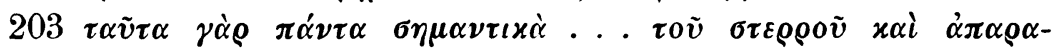

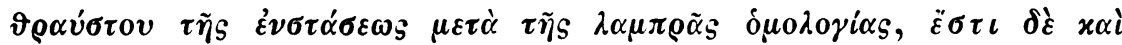

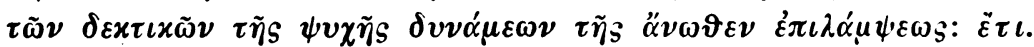

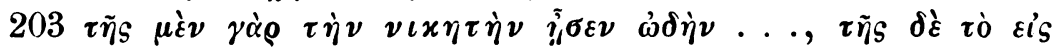

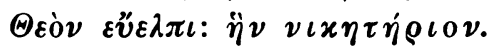

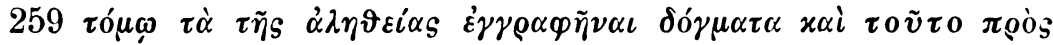

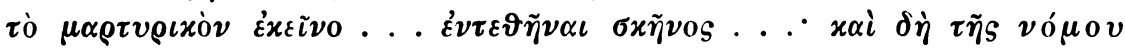

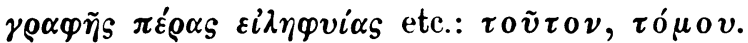

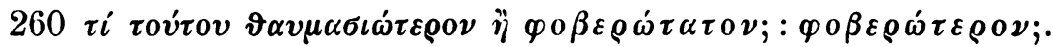

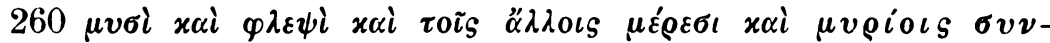

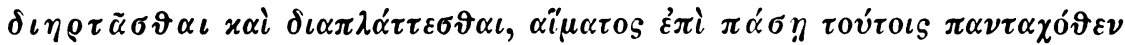

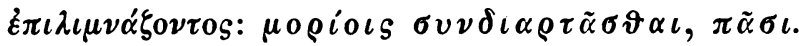

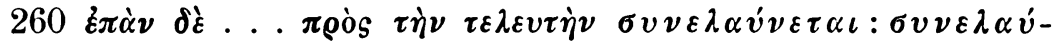
$\nu \eta \tau \alpha \iota$.

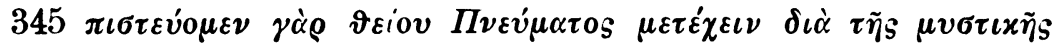

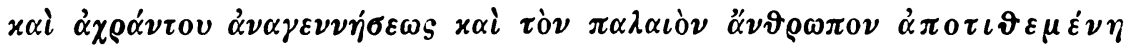

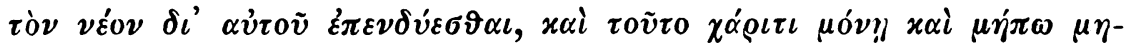

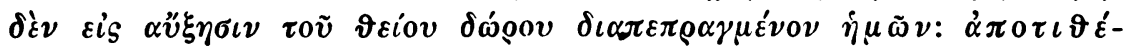
$\mu \varepsilon \nu \circ \iota, \dot{\eta} \mu \tilde{\nu}$.

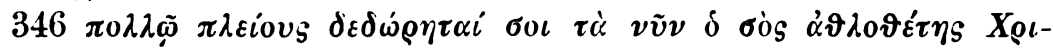

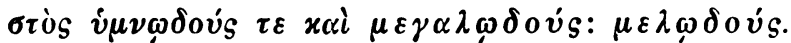

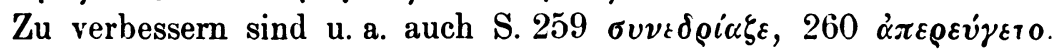
$\mathrm{Zu}$ lauterem Unsinn wird oft der Sinn des Verfassers durch die Interpunktion der $\mathrm{Hs}$, die leider in der Ausgabe treulich festgehalten ist.

Saloniki.

P. N. Papageorgin. 